\title{
X-Ray Emission from Broadcast Transmitters
}

\author{
E. Bruce Hunter - Voice of America \\ Route 1, Box 190 \\ Greenville, North Carolina 27834
}

\begin{abstract}
During the past few years, amplitude-modulated transmitters employing efficient pulse-width modulators have come into common use. These transmitters are reliable, relatively insensitive to vacuum tube characteristics, and offer excellent audio fidelity; however, their architecture requires supply voltages in the 20 to 30 kilovolt range which greatly enhances the production of ionizing radiation in the form of $x$-rays. Recent analysis by Voice of America indicates that these $x$-rays are being emitted in short, high-intensity bursts focused into broad radial beams. Part I of this paper reviews the general aspects of ionizing radiation of interest to communications engineers. Part II examines the spectral and time domain characteristics of $x$-ray radiation from transmitters, suggests a method for estimating fault condition radiation values, and discusses the findings of the Voice of America.
\end{abstract}

\section{PART I}

Nature of Ionizing Radiation

By definition, ionizing radiation is that wave and particulate radiation which is sufficiently energetic to overcome the binding forces holding atomic electrons to nuclei, and is thus capable of causing chemical changes in matter. Binding energy levels vary with the element but are typically on the order of five electron-volts (an electron-volt $(\mathrm{eV})$ being the kinetic energy acquired by an electron that has been accelerated through a potential of one volt). [1]

All electromagnetic radiation is emitted in photons or discrete quanta each of energy hc/lambda, where $h$ is Planck's constant $\left(6.62 \times 10^{-34}\right.$ joules-sec), $c$ the speed of light, and lambda the wavelength of the radiation. For electromagnetic radiation to be ionizing its photons must have minimum energies of about five electron-volts [2], and thus wavelengths of less than about 2000 angstroms (an angstrom being $10^{-10}$ meter). This corresponds to the upper ultraviolet light spectrum. The $\mathrm{x}$-ray spectrum (Figure 1) ranges from about 10 down to .01 angstroms, which corresponds to photon energies of 1200 to one million electron-volts, and frequencies of $3 \times 10^{17}$ hertz to $3 \times 10^{20}$ hertz [3].

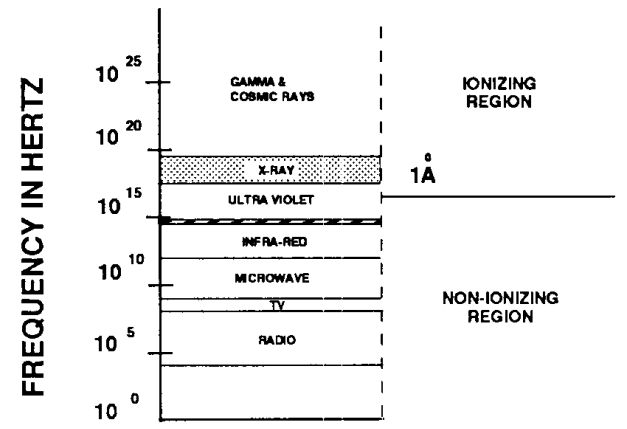

FREQUENCY SPECTRUM FIGURE 1

\section{Physics of X-ray Generation}

The majority of $\mathrm{x}$-ray emission from transmitting vacuum tubes occurs when speeding electrons are rapidly decelerated while passing through the strong electric fields that emanate from the positive charges in the nuclei of anode material atoms. An electron entering a metallic target may have its path bent around several different nuclei, at each point slowing down and emitting a photon of energy equal to the kinetic energy lost as it changes velocity (Figure 2). Such radiation is termed

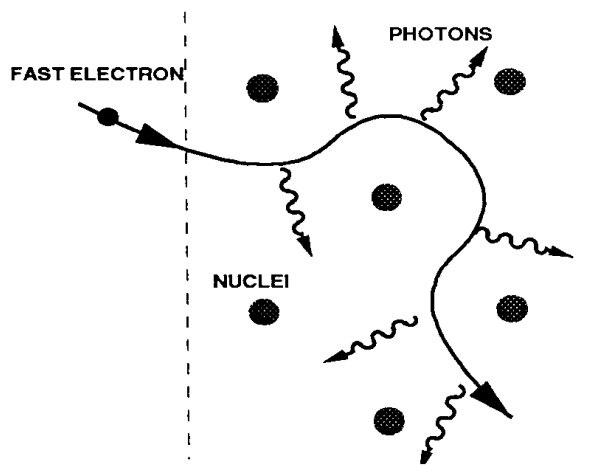

FIGURE 2

bremsstrahlung or slowing-down radiation. It has a broad, continuous distribution of wavelengths corresponding to energies from zero to a maximum numerically equal in electron-volts to the accelerating voltage. It is similar in spectral character to white noise except that the amplitude of the bremsstrahlung spectrum decreases in a straight line fashion with frequency (Figure 3). In practice, in passing through absorbing materials attenuation increases rapidly with wavelength causing the high-pass filtered characteristic shown by the solid line. This phenomenon is used

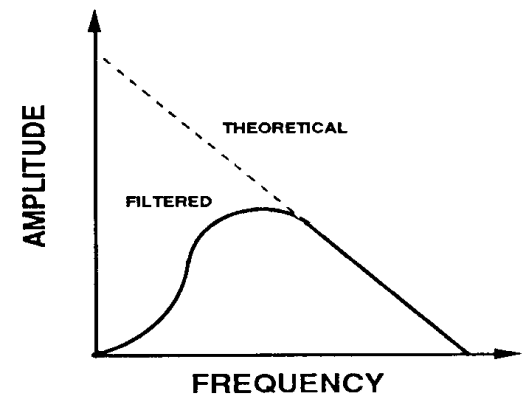

BREMSSTRAHLUNG SPECTRUM

FIGURE 3

U.S. Government work not protected by U.S. copyright 
in medical $\mathrm{x}$-ray equipment to remove the long-wavelength, lowenergy radiation that would otherwise be absorbed in the patient's skin, but would not contribute significantly to the radiographic image.

$\mathrm{X}$-rays are also generated when speeding electrons collide with the electrons in target material atoms (Figure 4). If an inner-shell electron is knocked free of its atom, the resulting unbalanced nuclear attraction will draw a replacement electron from an outer shell which causes a photon to be emitted with an energy equal to the difference in binding

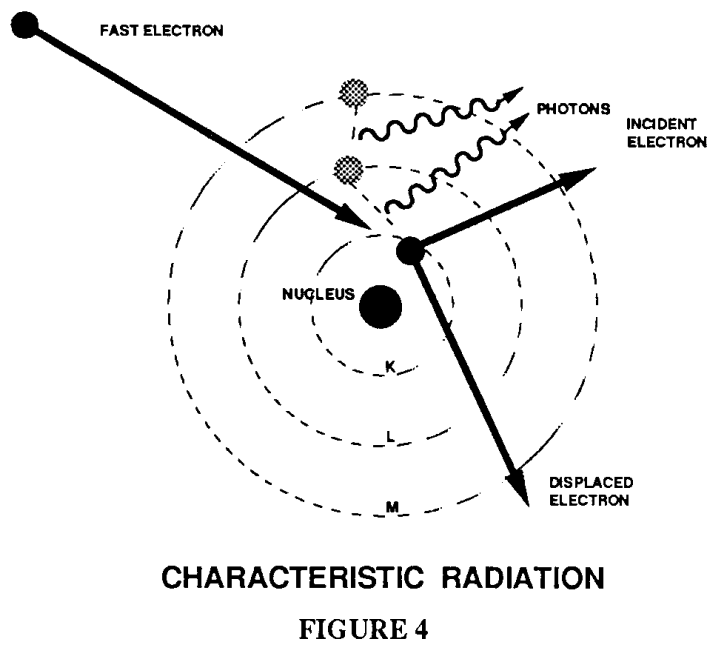

energics between the two electron shells. This is termed characteristic radiation because it occurs at well-defined frequencies that are characteristic of the target material and particular shell transition. Figure 5 shows the spectral shape of radiation from a tungsten target when the accelerating voltage is 100 kilovolts. Both bremsstrahlung and characteristic radiation are present. Typical high-pass filtering is shown.

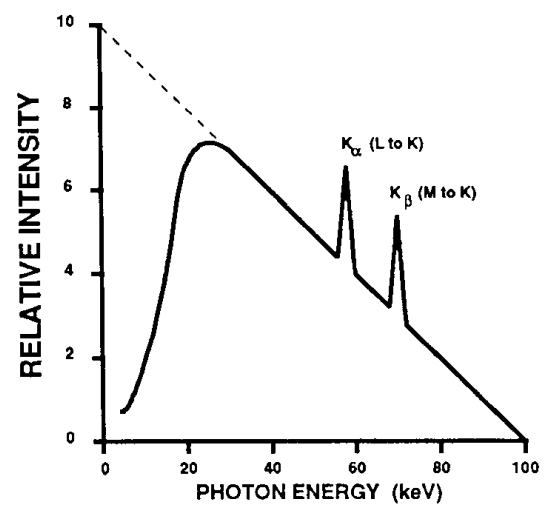

CHARACTERISTIC AND BREMSSTRAHLUNG RADIATION OF TUNGSTEN

FIGURE 5
The efficiency of conversion of electrical power to $\mathrm{x}$-ray frequency energy is approximately $9 \times 10^{-10}$ times the product of the accelerating voltage and the atomic number of the target material. For a 30 kilovolt supply, this is about $0.2 \%$ for tungsten and $0.08 \%$ for copper. Figure 6 shows how rapidly $\mathrm{x}$-ray emission increases as a function of accelerating voltage. The contours represent accelerating voltages of $20,30,40$, and 50 kilovolts respectively. Total $\mathrm{x}$-ray emission is proportional to the area under each curve. It varies approximately as the square of the applied voltage because it is proportional to the available direct current power times the efficiency factor discussed above.

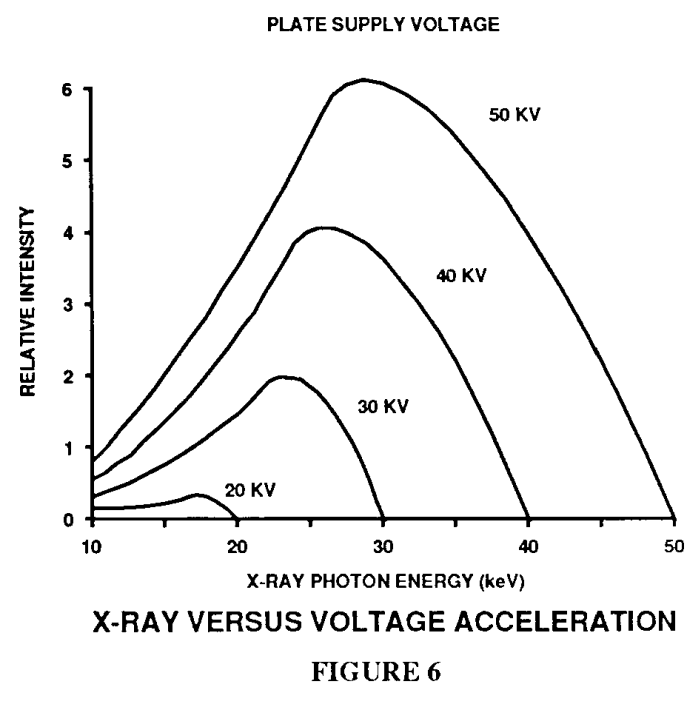

\section{Units of Measurement}

The commonly used units of measurement in x-ray physics are the roentgen, rad, and rem. These are modified by the customary scaling prefixes milli, micro, etc.

The roentgen is referred to as the unit of exposure. The term exposure is used in $\mathrm{x}$-ray physics to mean radiation intensity. The roentgen was originally defined as the level of radiation producing one electrostatic unit of ionization per cubic centimeter of air. Today it is defined in equivalent mass terms as the level of radiation producing $2.58 \times 10^{-4}$ coulomb of ionization of either polarity per kilogram of air. The roentgen is symbolized by the capital letter $\mathrm{R}$. Its equivalent value in power flux density units will be seen to vary with frequency.

The rad is an acronym for Radiation Absorbed Dose. It is a measure of the radiation absorbed in any kind of material including biological tissue. A dose of one rad is defined as the absorption of $100 \mathrm{ergs}$ of energy per gram of absorbing material. As can be seen from Figure 7, which shows the rads of absorption per roentgen for air, water, muscle, and bone as a function of photon energy, the number of rads absorbed is almost equal numerically to the exposure in roentgens, except for bone which absorbs radiation at about four times this rate. This similarity in absorption occurs because the probability of a photon interacting with an absorbing material is proportional to electron density, and this is approximately the same for air, water, and biological tissue. Because of its high calcium content, bone has about four times the electron density of air and water. The rad is symbolized with lowercase letters. 


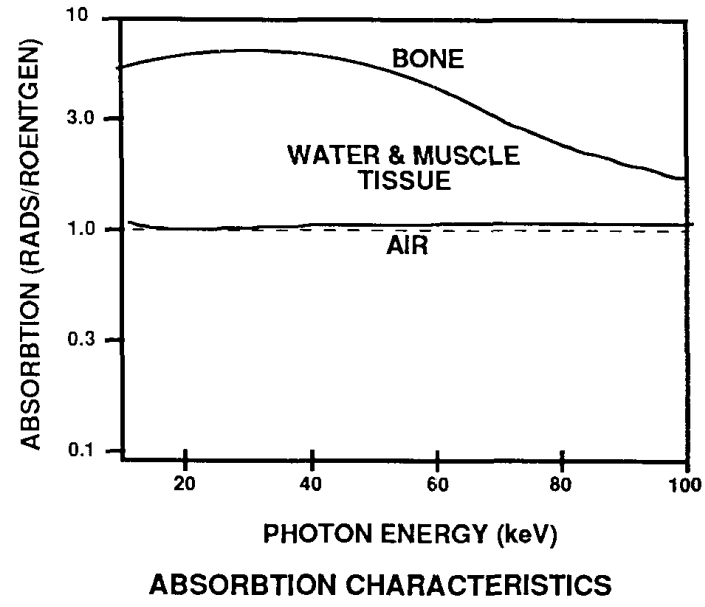

FIGURE 7

Because the rad is used as a measure of absorbed dose for all ionizing radiation including particulate radiation, a further adjustment is needed to account for the relative biological damage caused by absorption of the same number of rads of different types of ionizing radiation. For this purpose the unit rem, standing for Radiation Equivalent Man, was established and defined as equaling the rads absorbed times a numerical constant termed the Relative Biological Effectiveness (RBE). For $\mathrm{x}$-ray radiation, this factor is set equal to 1.0 [4]; therefore, for $\mathrm{x}$-rays from transmitters, the units of exposure and absorption, the three R's roentgens $(\mathrm{R})$, rads, and rems - are approximately equivalent numerically, even though they represent different physical quantities.

The SI units - gray which equals 100 rads and sievert which equals 100 rem are coming into common use and may ultimately replace the rad and rem.

Returning to the relationship between the roentgen and power flux density units, e.g., watts per square meter - surprisingly, the likelihood of interaction between photons and matter decreases with increasing photon energy or frequency. In other words the more energetic the photon, the greater the probability that it will simply pass through an absorber without interacting or causing damage. Figure 8 shows how the percentage of radiation absorbed by a one centimeter thickness of air rapidly decreases as incident photon energy increases.

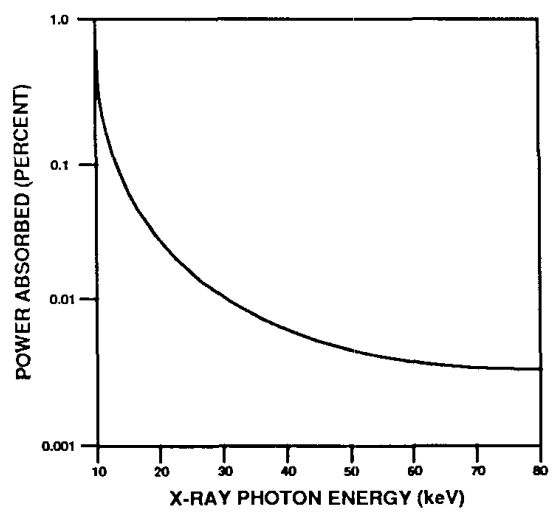

ABSORBTION IN AIR

FIGURE 8

As a result of the frequency-sensitive absorption factor shown in Figure 8 , the power flux density corresponding to an exposure of one roentgen per hour (and thus the absorption of 100 ergs per gram per hour in air) is caused to vary with frequency or photon energy as shown in Figure 9 for a volume of air exposed to $\mathrm{x}$-rays. A one roentgen per hour exposure level occurs when the power flux density is about $5 \times 10^{-10}$ watts per square meter for $10 \mathrm{keV}$ photons, and $4 \times 10^{-8}$ watts per square meter for $40 \mathrm{keV}$ photons.

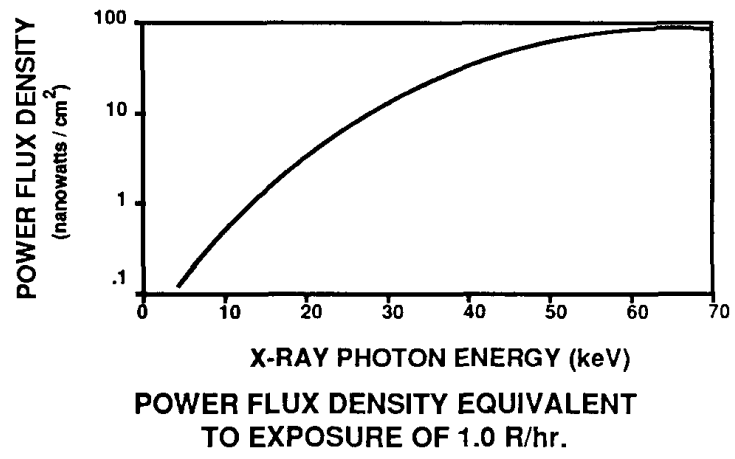

FIGURE 9

It is because of the way this frequency-sensitive absorption of $\mathrm{x}$-ray energy in air closely tracks the absorption in biological tissue that the roentgen is widely used in preference to power flux density units for radiation protection purposes. The roentgen serves to predict the number of rads (or ergs) biological tissue will absorb in a given level of exposure without requiring knowledge of the energy level of incident photons.

\section{Measuring Instruments}

The ionization chamber (Figure 10) is the fundamental instrument for measuring exposure according to the definition of roentgen. In a simple implementation, photons striking and ionizing air molecules create ion pairs that are separated and drawn to charged plates, and the resulting current is measured with a sensitive meter. The ionization chamber serves as a basic standard because its response is quite uniform; however, sensitivity is low, and it is not a good search tool for transmitters. Typical instruments have full-scale sensitivities ranging from about $0.5 \mathrm{mR} / \mathrm{hr}$ to $5 \mathrm{R} / \mathrm{hr}$.

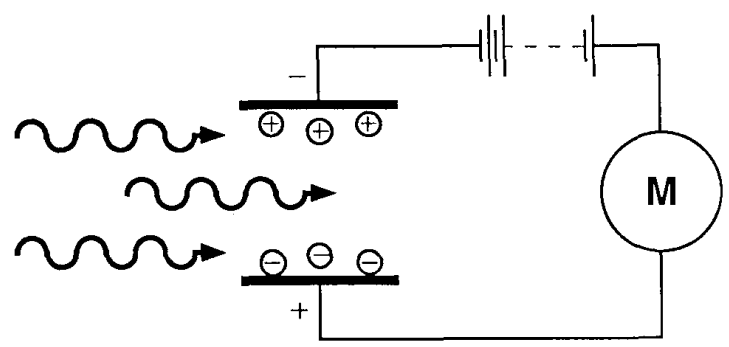

IONIZATION CHAMBER

FIGURE 10 
The Geiger/Mueller Counter (Figure 11) is similar to an ionization chamber except that its electrodes are arranged coaxially and electrified with a voltage sufficiently high so that single ionizations are multiplied through an av alanche process producing strong output pulses. Although rugged, relatively inexpensive, and typically about ten times more sensitive than the ionization chamber, the Geiger/Mueller counter is still somewhat insensitive. Typical full-scale sensitivities range from 0.1 to $100 \mathrm{mR} / \mathrm{hr}$. Geiger/Mueller counters are not recommended for transmitter applications because the long recovery time following an avalanche may cause the instrument to effectively saturate when emission is in the form of short bursts. Geiger/Mueller survey instruments sold for detection of radioactive contamination are generally designed for high-energy gamma rays, and their relatively thick windows may severely attenuate lower-frequency $\mathrm{x}$-ray photons from transmitters, causing $\mathrm{x}$-ray radiation levels to be understated.

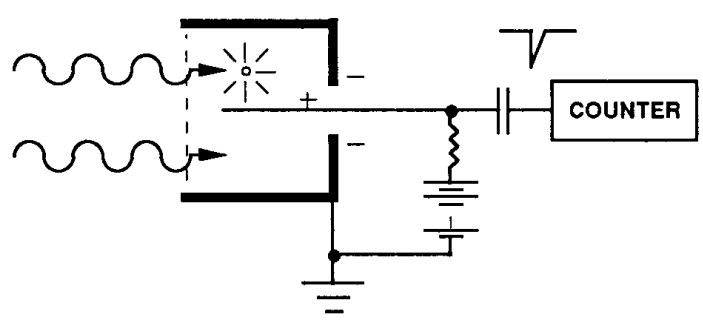

GEIGER/MUELLER COUNTER

FIGURE 11

The scintillation counter (Figure 12) is the most sensitive of the common radiation measuring instruments. In the typical portable instrument, a thallium-doped, sodium iodide crystal is coupled to a photomultiplier tube. Incident $\mathrm{x}$-ray photons cause the crystal to scintillate or give-off flashes of light. These are amplified and detected by the photomultiplier tube. Output pulse heights are proportional to the energy of incident photons. A typical portable instrument has scale ranges equivalent to 13,130 , and 1300 microroentgens per hour. Radiation exposures as low as a few microroentgens per hour can easily be detected.

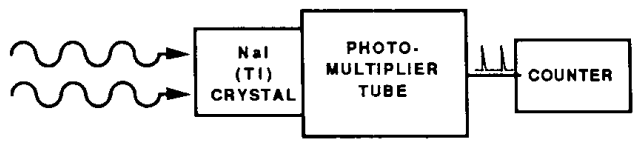

\section{SCINTILLATION COUNTER}

\section{FIGURE 12}

Radiation scalers (Figure 13) are analogous to the spectrum analyzers used at lower frequencies in that they provide information about the spectral distribution of ionizing radiation. Generally a scintillation probe is used as a radiation detector, and pulses from its output are sorted according to amplitude range into a series of separate channels for counting. Because pulse height is proportional to photon energy, and thus to frequency, the number of counts accumulated in each channel is an indication of the cumulative energy from waves of that energy or frequency range. Operation is similar to multi-channel, realtime audio spectrum analyzers.

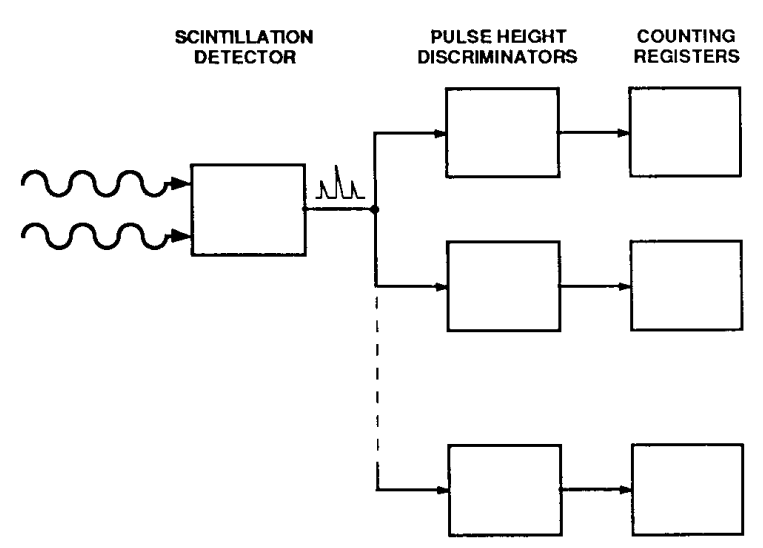

RADIATION SCALER

FIGURE 13

Lastly, there are various types of cumulative dosimeters that can be carried on the person or secured in fixed locations. These measure cumulative exposure, and utilize either a strip of film, which is later developed, a special plastic sensor, or an ion chamber and electroscope to integrate and record absorbed radiation. Typical units can detect exposures as low as 10 millirems per month, which is about one percent of the maximum permissible occupational dose. Dosimeters are usually read on a monthly or quarterly basis.

\section{Biological Effects of Ionizing Radiation}

Unlike non-ionizing radiation whose effects are believed to be largely thermal, ionizing radiation readily damages biological tissue. Many early radiologists lost fingers, hands, arms, and even their lives because they were unaware of the destructive power of the Roentgen Rays [5]. Ionizing radiation can induce leukemia and other forms of cancer in humans and animals, provoke mutation by damaging chromosomes, and cause skin burns, cataracts, loss of hair, and premature aging.

The commonly used model for assessing radiation-induced damage and limiting exposure levels is the Linear Dose Model (Figure 14a). This is based upon the Cumulative Dose Hypothesis which assumes that ionizing radiation doses received from all sources of radiation are cumulative over a lifetime; that there is no minimum exposure threshold below which damage will not occur; and that damage caused to the body from radiation exposure depends only on the cumulative dose received and not upon the rate at which it is received. Empirical evidence suggests that the body is able to repair some radiation damage over a period of time (although probably not that caused to chromosomal DNA chains). Very strong doses have clearly been found to have more effect than the same dose absorbed over a period of years. Although the actual case may be more like Figure $14 \mathrm{~b}$ or several other variations that have been proposed, for simplicity, the Linear Dose Model char acterized by Figure 14a continues to be the model most widely used for regulatory purposes.

To put radiation exposure into perspective, the U.S. public is thought to receive an average of about 100 millirems per year from natural background radiation, and about 90 millirems per year from medical and dental $\mathrm{x}$-rays. Watching a color television set adds about 0.5 millirem per year, and flying at 30,000 feet exposes one to about 0.5 millirem per hour, or about 3 millirems for a New York to $S$ an Francisco 

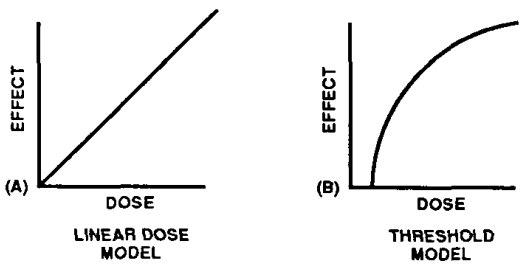

RADIATION MODELS

FIGURE 14

flight. In aggregate, a typical North American receives about 200 millirems per year from all sources.

At extreme levels of exposure, authorities estimate that fifty-percent of persons receiving a whole body dose of 450 rems or more over a period of minutes will die from radiation sickness within days to weeks. Note that even at this extreme dose level thermal effects are minimal. One writer has emphasized the extraordinary effectiveness of ionizing radiation for causing damage by calculating that body temperature would be raised by only about .001 degree Celsius from a rapidly received $450 \mathrm{rem}$ dose [6]. If this $450 \mathrm{rem}$ dose was received from a 30 $\mathrm{keV}$ monoenergetic source of $\mathrm{x}$-rays in one minute, the power flux density would be on the order of only 500 microwatts per square centimeter, which is equivalent to an electric field of about 40 volts per meter.

\section{Radiation Exposure Standards and Recommendations}

The Occupational Safety and Health Administration (OSHA) enforces $\mathrm{x}$-ray exposure limits in the work place [7], and generally sets the maximum dose workers may receive per calendar quarter as follows:

Whole body; head and trunk; active blood-forming Rems per calendar quarter organs; lenses of eyes; or gonads $-1.25$

Hands and forearms; feet and ankles18.75

Skin of whole body7.5

Persons under eighteen years of age are limited to ten-percent of these doses.

OSHA allows slightly higher doses in some cases, however the maximum dose to the whole body received during any calendar quarter must not exceed three rems, and the dose to the whole body, when added to the accumulated whole-body occupational dose, must not exceed $5(\mathrm{~N}-18)$ rems, where " $\mathrm{N}$ " equals the individual's age in years as of his last birthday.

Also of concern to employers, OSHA defines any area accessible to personnel in which the body might receive a dose in excess of 5 millirem during any one hour period as a Radiation Area. Such areas must be conspicuously posted with signs bearing the radiation symbol and the words CAUTION - RADIATION AREA.

These are only partial listings. OSHA's regulations for exposure of employees to ionizing radiation are found in Title 29, Subpart B, Code of Federal Regulations (CFR) Section 1910.96.
The Center for Devices and Radiological Health, which is a division of the U.S. Food and Drug Administration, regulates consumer products thatgenerate $x$-rays including television receivers. The current exposure rate limit for television receivers is 0.5 milliroentgen in one hour, measured at a distance of five centimeters from any point on the external surface of the set [8].

The Voice of America, the international broadcasting branch of the U.S. Information Agency, has applied basically this same specification to transmitting equipment being purchased for modernization of its stations.

\section{X-Ray Radiation Attenuation and Shielding}

At frequencies used for communications, conducting sheets function as shields by generating counteracting electromagnetic fields that reflect incident radiation. However because $\mathrm{x}$-ray waveleng ths are comparable to inter-atomic spacings, and because $\mathrm{x}$-ray photons can dislodge electrons in conductors, only a small amount of $\mathrm{x}$-ray energy is directly reflected [9]. $X$-ray radiation is attenuated by scattering, and by several physical processes that ultimately reduce it to heat as a result of multiple interactions in the absorbing material [10]. The loss of exposure or power flux density in passing through an $\mathrm{x}$-ray absorber follows a negative exponential law:

(1)

$$
I_{x}=I_{0} e^{-\mu x}
$$

where $I_{0}$ is the incident power flux density, $I_{x}$ the fraction remaining after passing through thickness $(x)$ of the absorbing material, and $\mu$ the linear attenuation coefficient for the material at a specific wavelength. This implies the attenuation in $\mathrm{db}$ is:

$$
A(d b)=4.34 \mu x
$$

Tabulations in reference materials are often found in terms of $\mu /$ tho, the mass attenuation coefficient. These are easily converted to $\mu$ by multiplying by rho, the mass density of the absorbing material.

The equations above are predicated upon monoenergetic sources of photons, a condition seldom found in practice. Nevertheless, useful calculations can be made by employing estimates of the mean photon energy of an x-ray beam. Because the linear attenuation coefficient for most materials increases almost as the cube of $x$-ray wavelength, it is possible to estimate the mean energy of an $\mathrm{x}$-ray beam by inserting metal plates of various thickness in the beam until the intensity is reduced by fifty percent. The required thickness is termed the half-value layer and is tabulated in reference books as a function of mean energy level. Alternately, an arbitrarily thick piece of metal that produces an attenuation of about $3 \mathrm{db}$ can be used and $\mu$ computed as:

$$
\mu=\frac{1}{x} \ln \left(\frac{I_{0}}{I_{x}}\right)
$$

Once $\mu$ is known, the mean photon energy in $\mathrm{keV}$ can be found in a table for the appropriate metal based upon its thickness. Attenuations much greater than $3 \mathrm{db}$ should be avoided because the previously mentioned "high-pass filtering" will skew the indicated effective energy upward.

Figure 15 shows the attenuation of a one millimeter thickness of common metals as a function of photon energy. Notice that in addition to being a good RF shielding material, copper also is a fairly effective $\mathrm{x}$-ray absorber. Shell transition effects termed absorption edges cause attenuation to drop sharply at some frequencies. These do not occur for the range of energy and materials shown in Figure 15. Designers should consult tabulations in the references for other energy ranges. 


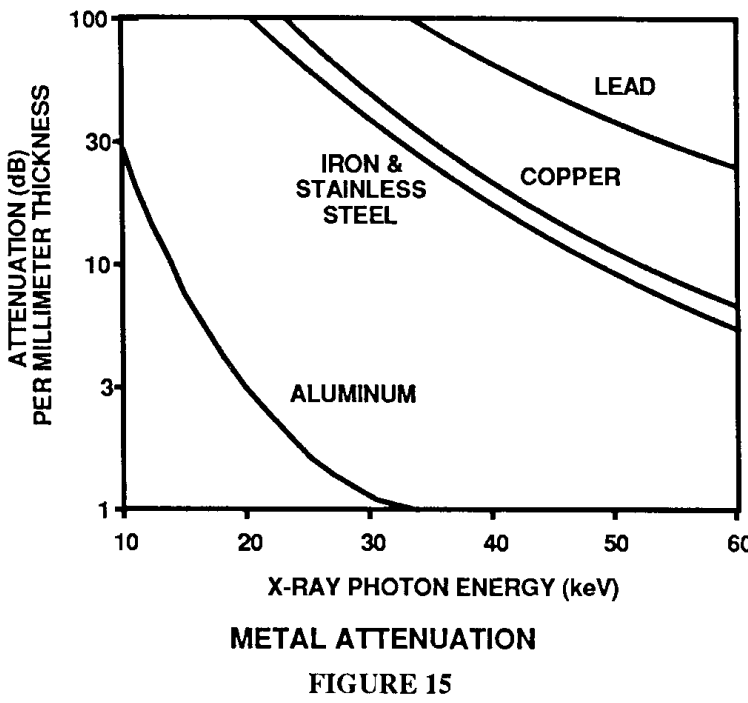

Many special protective and shielding products are available from suppliers of radiological equipment. A lead sheet of one and one-half millimeter thickness (about one-sixteenth inch) is specified for many medical radiological applications, and lead sheets of this thickness, conveniently laminated to vinyl or other decorative and protective coatings, are readily available.

Glass with a high lead content is available for use in viewing windows, and as a shield for troubleshooting. A commonly used product is about one-quarter inch thick and has an attenuation equivalent to a one and one-half millimeter thick lead sheet.

\section{PART II}

\section{Spectral Significance of $\mathrm{X}$-ray Radiation}

The spectral characteristics of $\mathrm{x}$-ray radiation from transmitters offer an insight to tube operating conditions and may identify defective units. The upper energy limit of the spectrum may reveal the presence of otherwise unanticipated high voltage transients. The mean photon energy of an $x$-ray beam is typically one-third to one-half of its maximum photon energy, which, in turn must be numerically less in electron-volts than the tube's peak anode voltage. With this in mind, examine Figure 16 which shows the $x$-ray emission spectrum of a pulsewidth modulator tube operating from a 30 kilovolt direct current power supply. This was recorded with a scintillation probe and 550 channel scaler system in which photon energy levels are proportional to the scaler's channel numbers. The mean energy of the distribution shown is about $30 \mathrm{keV}$ (channel 275) which implies that the highest energy photons (channel 412) have an energy of about $45 \mathrm{keV}$. This in turn reveals that a peak voltage in the neighborhood of 45 kilovolts is occurring-a significant finding because high voltage components in this modulator have been prone to fail.

Study of the spectra of $\mathrm{x}$-ray radiation from high voltage vacuum tubes may prove to be an effective means of determining the magnitude of peak voltages occurring in transmitter circuitry. This technique can be employed under dynamic operating conditions without significantly affecting the waveforms being measured. Commonly used probes and voltage dividers add capacitance to the circuits being observed, which increases the rise and fall times of waveforms, and generally decreases the peak value of transient voltages.

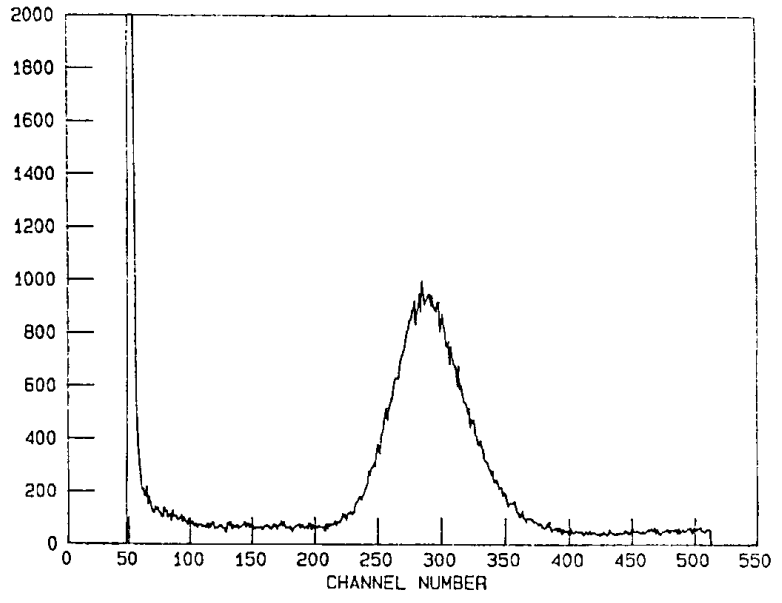

\section{RADIATION FROM TRANSMITTER MODULATOR FIGURE 16}

Time Domain Characteristics of Modulator X-ray Radiation

Figure 17 symbolizes the calculated time domain characteristic of a pulse-width modulator tube's x-ray emission. As can be seen, x-ray emission occurs in short bursts during the transitions of the modulator waveform. When the modulator tube is cut-off, $\mathrm{x}$-ray radiation results only from electrons spontaneously emitted from the grids. Likewise, when the tube is saturated, virtually no x-ray emission occurs. It is during the finite period spent switching-off, while the tube passes through the active portion of its plate characteristic, and when current still flows with nearly full voltage across the tube, that radiation is the most severe. X-ray emission then becomes insignificant as current ceases. A smaller burst occurs when the tube is switched-on. Observations with a scintillation probe and oscilloscope appear to empirically confirm theoretical calculations by showing that $\mathrm{x}$-ray radiation is occurring in bursts during the transitions of the modulator waveform, and by showing that this radiation is more severe when the tube is switchingoff than switching-on. The relatively-long recovery time constant of the scintillation probe that was employed prevented confirmation of the detailed waveforms of the bursts. Additional studies with a more quickly responding detector are desirable.

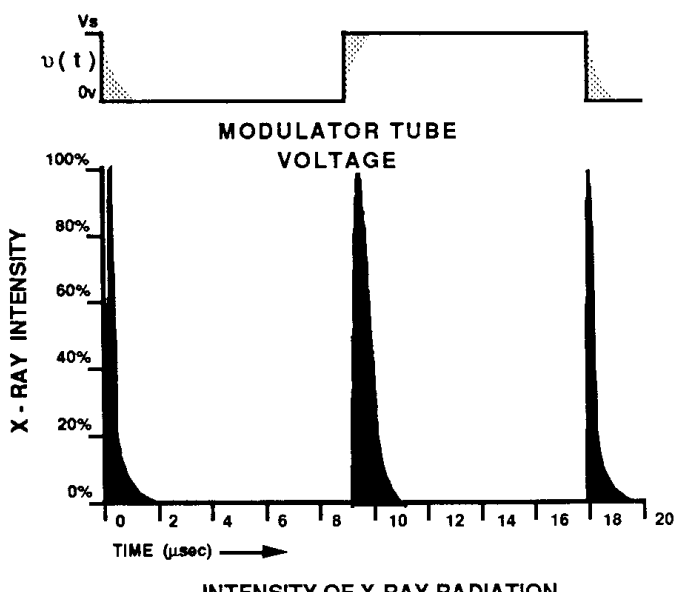

INTENSITY OF X-RAY RADIATION

MODULATOR TIME DOMAIN CHARACTERISTICS

FIGURE 17 
Class $C$ radio frequency amplifiers would be expected to exhibit similar time-domain $\mathrm{x}$-ray emission characteristics, with $\mathrm{x}$-rays being emitted in bursts when the tube passes through the active region of its plate characteristic.

The significant results observed here are that $x$-ray radiation is occurring in short, high-amplitude bursts which may be more damaging than the equivalent exposure received at a constant rate. Also, radiation measuring instruments may become saturated during the bursts and understate true average radiation levels. Finally, fault condition radiation levels, where large currents flow for significant periods of time with high voltages across tubes, may be far higher than average values. These may induce ionization in nearby components, and might be hazardous to personnel if protective shielding is removed or is inadequate.

As $\mathrm{x}$-ray emission varies directly with tube plate current, and as explained in Part I approximately as the tube voltage squared, an empirical equation (Equation 4) can be written relating the average radiation rate observed at a test point to an arbitrary constant $(k)$ times the normalized time integral of the product of the tube's instantaneous voltage ( $v$ ) squared times its current (i). If this integral can be evaluated, multiplicative constant $(k)$ can be calculated. (k) represents a combination of the efficiency of the $x$-ray generation process, the geometric directivity of radiation, and the fractional transmission of radiation through the wall of the tube. $(X)$ in Equation 4 represents the average radiation from the tube in roentgens-per-hour as observed at the point of interest. ( $T$ ) is the time period being considered.

$$
X=k \frac{1}{T} \int_{0}^{T} v^{2}(t) i(t) d t
$$

As Equation 4 can be evaluated for rectangular waveforms which rise and decay exponentially, the instantaneous voltage across a pulse width modulator tube was modeled with exponential equations based upon the simplified circuit of Figure 18. Actual measured rise and fall times $\tau_{2}$ and $\tau_{1}$ were used for purposes of calculation. The current waveform was calculated with the assumption that the tube's load was purely resistive. The following equations were used in which $\left(V_{s}\right)$ represents the available direct current power supply potential, $(R)$ the equivalent load resistance, and $v(t), i(t)$ the instantaneous values of voltage and current. $(T)$ is the overall length of the modulator waveform's period.

$$
v(t)=V_{s} e^{-\frac{t}{\tau_{1}}}: 0<t \leq t,
$$

(tube voltage while switching-on)

$$
v(t)=V_{2}\left(1-e^{-\frac{t}{\tau_{1}}}\right): t_{1}<t \leq t_{2}
$$

(tube voltage while switching-off)

$$
i(t)=\frac{V_{s}}{R}\left(1-e^{-\frac{t}{\tau_{2}}}\right): 0<t \leq t_{1}
$$

(tube current while switching-on)

$$
i(t)=\frac{V_{s}}{R} e^{-\frac{t}{\tau_{2}}}: t_{1}<t \leq t_{2}
$$

(tube current while switching-off)

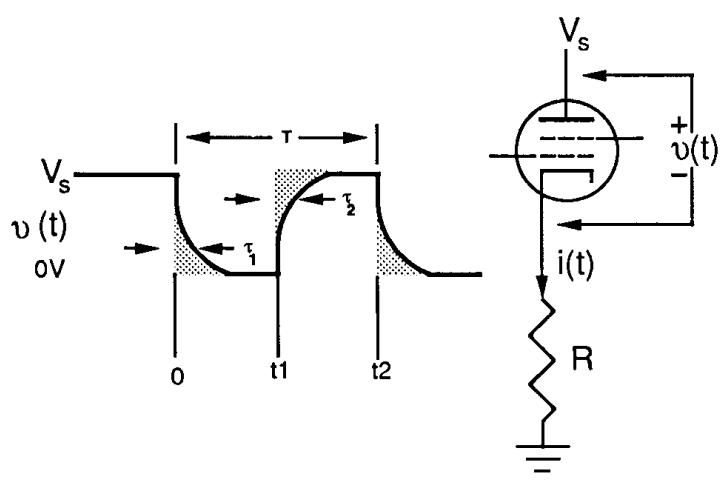

MODEL FOR X-RAY CALCULATION

FIGURE 18

For the model of Figure 18, and Equations 5 through 8, Equation 4 can be written:

$$
\begin{aligned}
X= & k \frac{1}{T} \frac{V_{s}^{3}}{R}\left\{\int_{0}^{t}\left(e^{\frac{-2 t}{\tau_{1}}}-e^{\frac{-3 t}{\tau_{1}}}\right) d t\right. \\
& \left.+\int_{t_{1}}^{t_{2}}\left(e^{\frac{-t}{\tau_{2}}}-2 e^{\frac{-2 t}{\tau_{2}}}+e^{\frac{-3 t}{\tau_{2}}}\right) d t\right\}
\end{aligned}
$$

Ignoring insignificant terms:

$$
X=k \frac{1}{T} \frac{V_{2}^{3}}{R}\left(\frac{\tau_{1}}{6}+\frac{\tau_{2}}{3}\right)
$$

Assuming equal rise and fall times $\tau_{2}$ and $\tau_{1}$, it is interesting to note from Equation 10 that the tube produces twice as much total radiation in switching-off as in switching-on (see Figure 17). This is because when switching-off, nearly full voltage is present across the tube during the period in which the current is slowly decaying asymptotically. When switching-on, the high voltage portion of the tube characteristic is passed-through quickly and asymptotic decay occurs as the tube approaches saturation.

The term switching-on is used in this paper to label the point at which the tube begins to conduct and plate current begins to flow; at this time the voltage across the tube falls from a high to a low value.

To gauge the accuracy of the exponential model, the product of the instantaneous values of voltage and current was integrated over the switching period to calculate the tube's power dissipation. This was compared with the transmitter manufacturer's calorimetric measurement and found to be in good agreement.

Solving Equation 10 for $(k)$ :

$$
k=\frac{6 R T X}{V_{3}^{3}\left(\tau_{1}+2 \tau_{2}\right)}
$$

With $(k)$ determined, it can be used to estimate fault condition radiation values. In the case calculated, this constant turned out to be about 1.5 $x 10^{-11}$ roentgens-per-hour for each volt-squared-ampere, or for the likely range of fault conditions, on the order of several hundred times 
the value of emission observed under normal conditions. Peak x-ray generation potential under fault conditions is primarily limited by the emission capability of the tube, the energy stored in the power supply filter capacitance, and the resistance and inductance of connecting leads.

The average radiation rate $X_{f}$ found under fault conditions can be calculated as:

$$
X_{f}=k \frac{1}{T} \int_{0}^{T} v^{2}(t) i(t) d t
$$

where $v(t)$ and $i(t)$ are arbitrary functions of time. The same value of $(k)$ applies when the tube type is used as an RF power amplifier, or for other purposes, because it is a function of tube geometry, and not of the particular voltage and current waveforms.

By assuming constant values $\mathrm{V}, \mathrm{I}$ for voltage and current during fault conditions, radiation can be estimated simply as:

$$
X_{1}=k V^{2} l
$$

It would be useful if tube manufacturers provided an approximate value for the aforementioned constant allowing estimation of the worst-case, peak value of $\mathrm{x}$-ray radiation likely to be found at a distance of one meter from any point on the surface of their products. This would be only an approximation because emission levels and radiation patterns tend to vary somewhat with individual tubes.

A similar computational technique could be used to calculate peak-toaverage $\mathrm{x}$-ray emission ratios for use in determining whether a given measuring instrument might saturate.

\section{Irradiation of Transmitter Components}

A European manufacturer of vacuum capacitors [11] has recently suggested that $\mathrm{x}$-ray radiation from vacuum tubes might be playing a role in the premature failure of vacuum capacitors. The suspected mechanism is the photoelectric effect in which incident photons cause electrons to be dislodged from copper atoms, which causes internal emission and facilitates arcing between adjacent capacitor plates. This manufacturer indicated that as a test, $\mathrm{x}$-ray shielding has been installed around a failure-prone capacitor position in a transmitter. No results have been announced as yet.

It is possible that $\mathrm{x}$-ray radiation (particularly that occurring under fault conditions) is contributing to the failure of other transmitter components such as the metalized, plastic dielectric, screen bypass capacitors sometimes built into the sockets of high-frequency power amplifier tubes. Premature failures might be occurring either because of ionization caused by instantaneous $x$-ray bursts that result from fault conditions, or as a consequence of long-term chemical changes brought about by incident radiation. More study is required.

\section{VOA Experience}

Several years ago, as part of its worldwide Modemization Program, VOA purchased four contemporary-design 500 kilowatt transmitters from four leading manufacturers to assess the international state-of-theart in transmitter technology. These transmitters were subjected to exhaustive testing, including the measurement of $x$-ray radiation [12].
At first, a relatively-insensitive ionization chamber meter was used and little radiation in excess of the desired limit was detected. Later, testing with a more sensitive instrument showed that the desired limit was being frequently exceeded, and a viewing window in one transmitter was replaced with $\mathrm{x}$-ray absorbing lead glass.

Subsequently, a sensitive scintillation counter designed for $\mathrm{x}$-ray radiation in the range up to $60 \mathrm{keV}$ was purchased and used to map contours on the transmitter cabinets. Areas where a $0.5 \mathrm{mR} / \mathrm{hr}$ level was exceeded are shown in Figures 19 (a) through (f). Based on these findings, some manufacturers were asked to install additional $\mathrm{x}$-ray shielding in their products.

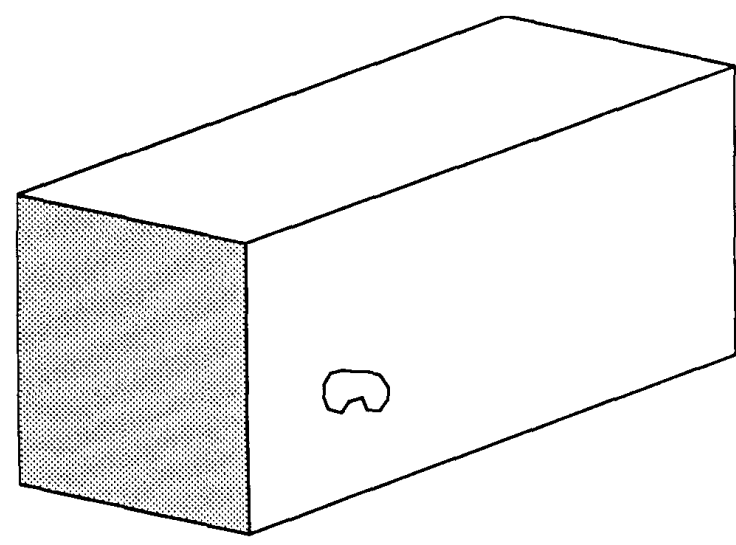

(a) PULSE - WIDTH MODULATOR

FIGURE 19A

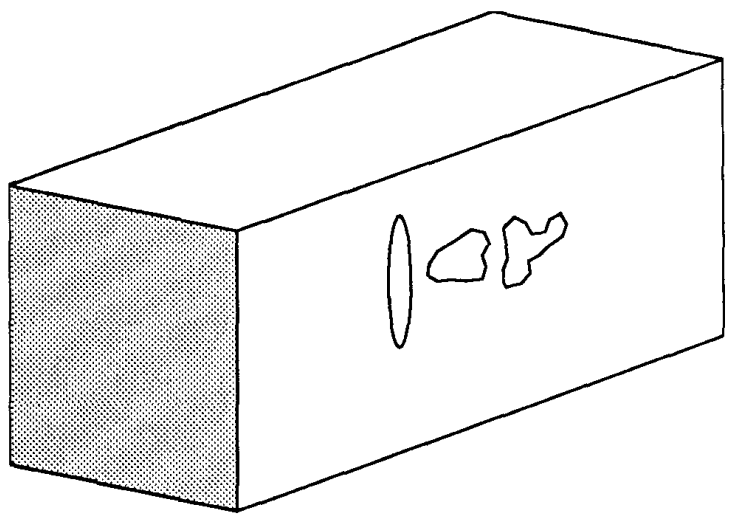

(b) PULSE WIDTH MODULATOR

FIGURE 19B 


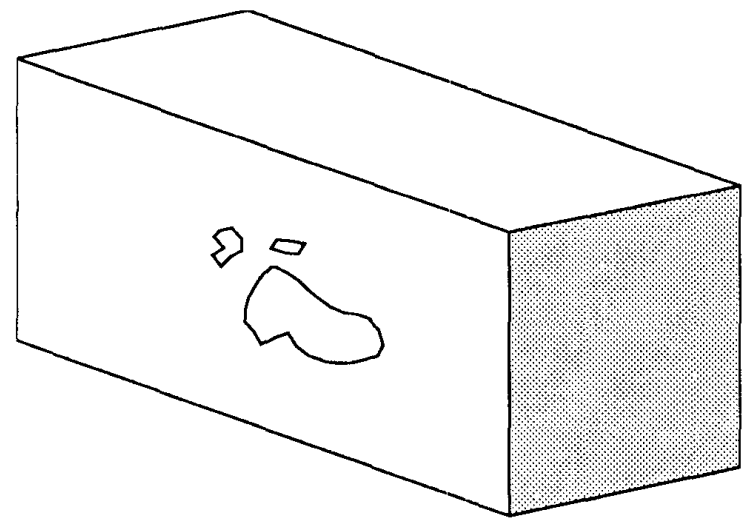

(c) PULSE - WIDTH MODULATOR FIGURE 19C

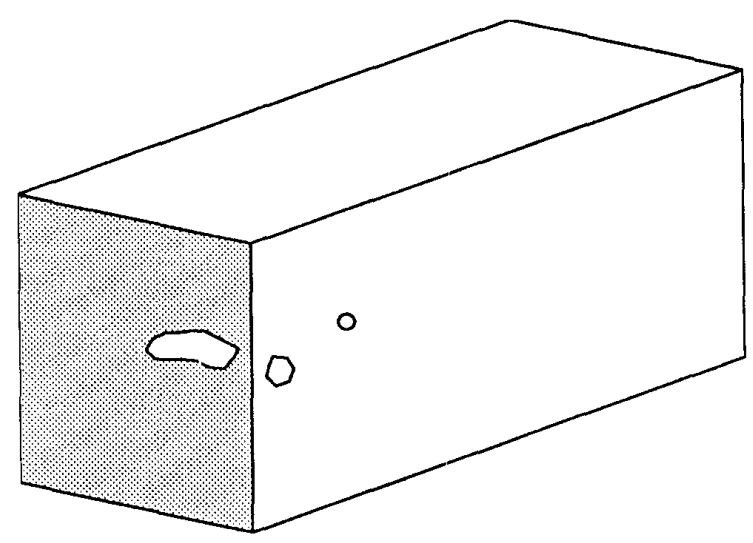

(d) RF POWER AMPLIFIER

FIGURE 19D

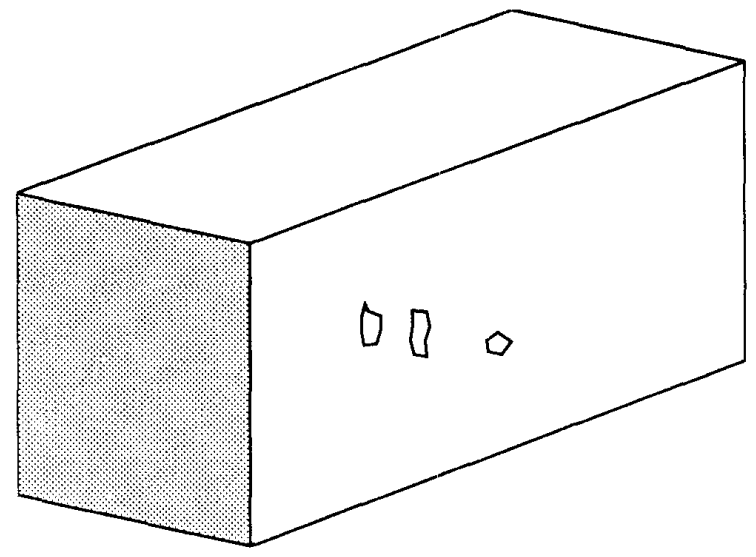

(e) PULSE WIDTH MODULATOR FIGURE 19F

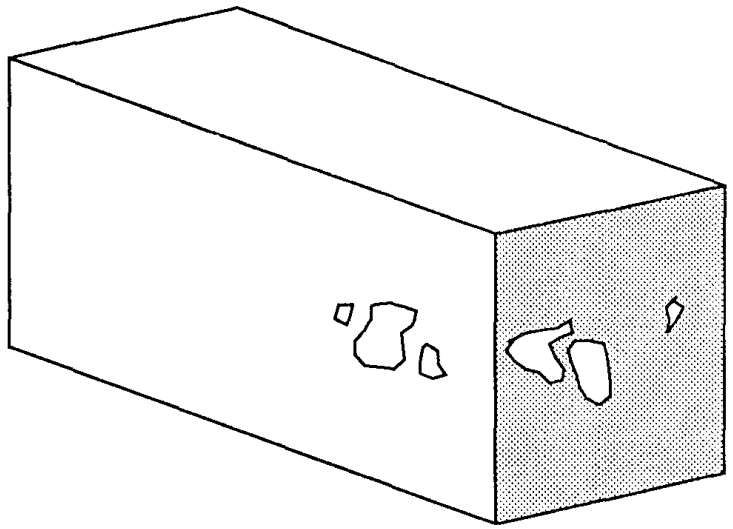

(f) RF POWER AMPLIFIER FIGURE 19F

The scintillation detector employed had a sensitivity on the order of one thousand times the desired $0.5 \mathrm{mR} / \mathrm{hr}$ limit. This sensitivity and the instrument's audible "clicker" allowed the operator to quickly locate the sources of radiation without the aid of an indicating meter. Because the $\mathrm{x}$-ray radiation was found to be emitted in concentrated beams, locating it with a less-sensitive, integrating-type meter, which must be held in each position for five or more seconds to obtain a reading, would have required a very disciplined, time-consuming, search pattern.

Only small amounts of $x$-ray radiation were detected from transmitters operating with plate potentials in the 12 to 15 kilovolt range, even though the peak plate voltage of one type reached 24 kilovolts during modulation peaks.

\section{Conclusions}

Operators and maintenance technicians who work with transmitters employing voltages in excess of 20 kilovolts should be cautioned that doors and radiation shields should not be opened or removed for observation of arcing, because it is under just such fault conditions that the most serious radiation is likely to occur. Particularly with 500 kilowatt transmitters, $x$-ray radiation surveys are recommended following tube changes, or whenever transmitter operating conditions appear to have changed. Employees working with such equipment should be acquainted with the fundamentals of ionizing radiation physics and the hazards associated with exposure to this form of radiation.

Sensitive scintillation counters, designed for detection of $x$-rays in the 10 to $60 \mathrm{keV}$ range, are the best search instruments, both for examining new equipment, and performing routine maintenance checks. Less sensitive ion chamber instruments are preferred for making final, quantitative measurements for regulatory compliance purposes because they have more accurately known response characteristics, and are less likely to saturate in pulsed applications. At least one manufacturer offers a high-sensitivity, ion chamber instrument that has been carefully shielded to prevent radio frequency pickup.

The presence of high peak voltages, which may be causing failure of circuit components, will likely be revealed by observing the spectral characteristics of emitted $x$-ray radiation. This can generally be done without disturbing circuit operating conditions. 
Consideration should be given to the possibility that $\mathrm{X}$-ray emission may be contributing to the premature failure of materials and components that are located in the proximity of high power vacuum tubes.

Acknowledgements

The author wishes to thank Professor James Gaiser and Dr. Daniel Sprau of East Carolina University for making the spectral measurements of emission from the transmitter modulator, VOA Field Engineer James Nelson who made the time domain $\mathrm{x}$-ray emission tests reported in this paper, and VOA engineers Satish Bhatia, Marion Hales, and Robert Wilson who permitted their transmitter $\mathrm{x}$-ray radiation contour diagrams to be included in this paper.

Notes

[1] The present discussion focuses on x-ray radiation. Gamma radiation is identical in character but originates in the nuclei of atoms.

[2] Varying energy levels are cited for the point at which electromagnetic radiation becomes ionizing. Ionization is a stochastic process, and although the probability that a $5 \mathrm{eV}$ photon will dislodge an electron with a binding energy of $5 \mathrm{eV}$ is zero, it becomes finite and grows rapidly as the incident photon energy increases. For this reason values of 10 to $100 \mathrm{eV}$ are sometimes stated as the threshold of ionizing radiation.

[3] The range given for $x$-rays is typical of the radiation from medical $\mathrm{X}$-ray equipment. X-ray tubes operating at higher voltages, betatrons and proton accelerators can produce $\mathrm{x}$-rays with photon energies exceeding $12.4 \mathrm{MeV}$ corresponding to wavelengths of less than .00001 angstrom. (See Reference 2.)

[4] RBE factors for other forms of ionizing radiation are: $x$-rays, gamma rays, and electrons $=1$; Neutrons $=3$ to 10 depending on energy level; and heavy particles $=1$ to 20 . For further information, see National Council on Radiation Protection and Measurements (NCRP) Report 39.

[5] See, for example, Percy Brown M.D., American Martyrs to Science through the Roentgen Rays. Springficld, Illinois: Charles C. Thomas, 1936.

[6] John W. Gofman, Radiation and Human Health. San Francisco: Sierra Club Books, 1981, pp. 52-53.

[7] The U.S. Nuclear Regulatory Commission licenses users of radioactive materials and reactor products. NRC regulations are found in Title 10 Code of Federal Regulations, Parts 19 and 20. These regulations would not normally apply to broadcast equipment.

[8] Regulations for $\mathrm{x}$-ray emission from television receivers are found in Title 21 Code of Federal Regulations, Part 1000,

Subpart J. The Center for Devices and Radiological Health is a unit of the U.S. Food and Drug Administration.

[9] Effective reflectors for $\mathrm{X}$-rays have only recently been constructed See D.L. Matthews and M.D. Rosen, "Soft X-ray Lasers," Scientific American, pp .90, Dec. 1988

[10] See any text on $\mathrm{x}$-ray physics for a discussion of the photoelectric effect, Comptoneffect, and Thomsonscattering, the physical mechanisms responsible for the attenuation characteristics discussed.

[11] Comet AG, Waldeggstrasse 72, CH-3097 Liebefeld-Bern, Switzerland. Comet produces vacuum capacitors and $\mathrm{x}$-ray tubes.
[12] Discussed in G.A. Berman and T.R. Garlington, "The Evaluation of $500 \mathrm{~kW}$ Shortwave Transmitters at the Voice of America," IEEE Transactions on Broadcasting, June 1988.

\section{References}

1. Copies of the Code of Federal Regulations and other Federal Government publications currently in print can be obtained from the Superintendent of Documents, U.S. Government Printing Office, Washington, DC 20402

2. Where it can be found, a complete source of $x$-ray reference data is the Handbook of Radiological Health, which was published annually by the U.S. Department of Health Education and Welfare through 1972. A currently published, updated, similar publication The Health and Physics Radiological Health Handbook, along with Supplement 1 (1986), is offered by Nucleon Lecturn Associates, 2919 Olney-Sandy Spring Road, Suite D, Olney, MD 20832.

3. The International Commission on Radiation Units and Measurements (ICRU) publishes a number of reports relating to ionizing radiation. Of particular interest is "Report 17,"Radiation Dosimetry: $X$-Rays Generated at Potentials of 5 to $150 \mathrm{KV}$, from which the absorption coefficients for air, water, tissue and bone used in this paper were taken. The mailing address is ICRU Publications, 7910 Woodmont Avenue, Suite 800, Bethesda, MD 20814.

4. The National Council on Radiation Protection and Measurements (NCRP) is chartered by the U.S. Congress and also offers many publications related to both ionizing and non-ionizing radiation. The mailing address is NCRP Publications, 7910 Woodmont Avenue, Suite 1016, Bethesda, MD 20814.

5. The National Institute of Standards and Technology (formerly the National Bureau of Standards) has published many handbooks containing radiological data. These include recommendations of the ICRU. Publications in print are available through the Superintendent of Documents, U.S. Government Printing Office, Washington, DC 20402

6. Many texts on various aspects of radiation physics are available; the following are represertative:

Anderson, D.W.Absorption of Ionizing Radiation. Baltimore: University Park Press, 1984

Dyson, N.A.X-RaysinAtomic and Nuclear Physics. London: Longman, 1973. This is an intermediate level text covering $X$-ray interactions in matter. Its appendix contains an extensive collection of empirical mass attenuation coefficient data.

Hendee, W.R. Medical Radiation Physics, Second Edition. Chicago \& London: Yearbook Medical Publishers, 1979.

Khan, F.M. The Physics of Radiation Therapy. Baltimore/London: Williams \& Wilkins, 1984

Sorenson, J.A. and Phelps, M.E. Physics in Nuclear Medicine. Orlando, New York: Harcourt Brace Jovanovich, 1987.

Tsoulf anidis, N. Measurement and Detection of Radiation. New York: McGraw-Hill, 1983.

7. Gofman's book referenced in [6] is written for the layman and provides a clear explanation of the biological hazards of ionizing radiation. It contains many citations of the data upon which current radiation exposure standards are based. 